\title{
Informed Consent - Balance zwischen Patientenschutz und Forschungsinteressen
}

\author{
Forschungsethische Debatten haben sich in den letzten Jahren häufig der Frage ge- \\ widmet, ob Ausnahmen bei der strikten Forderung nach individueller Einwilligung \\ des Patienten zulässig oder gar geboten sind. Einige Studiendesigns mit solchen Aus- \\ nahmeregelungen werden im Folgenden zur Diskussion vorgestellt.
}

\section{Margrit Fässler, \\ Nikola Biller-Andorno}

Institut für Biomedizinische Ethik, Universität Zürich

Es bestehen keine Interessenbindungen der Autorinnen. Die Arbeit wurde durch Fördermittel der Robert-BoschStiftung unterstützt.

a Für die Zusammenstellung in Kasten 1 wurden die relevanten internationalen Dokumente (Nürnberger Kodex, Deklaration von Helsinki, GCP und Bioethik-Konvention des Europarates) herangezogen.

\section{Korrespondenz:}

Dr. med. Margrit Fässler Institut für Biomedizinische Ethik

Universität Zürich

Zollikerstr. 115

CH-8008 Zürich

Tel. 0446348381

Fax 0446348389

faessler@ethik.uzh.ch

www.ethik.uzh.ch/ibme.html

\section{Einleitung}

Ethische Richtlinien wie die Deklaration von Helsinki und nationale Gesetze regeln die Einholung einer Einwilligung nach Aufklärung (Informed Consent) bei Studienteilnehmern. Dies ist zum Standard geworden, so dass sich heutzutage nicht die Frage stellt, $o b$ aufgeklärt wird, sondern wie. In bestimmten Situationen, vor allem bei randomisierten kontrollierten Studien, kann es schwierig sein, über bestimmte Studiendetails aufzuklären, weil dies zu verzerrten Ergebnissen (biases) führen kann und so die Verlässlichkeit von Forschungsergebnissen beeinträchtigt wird. Es stellt sich dann die Frage, ob es in solchen Situationen zulässig ist, den Teilnehmenden bestimmte Details über die Studie nicht mitzuteilen oder dies zu einem späteren Zeitpunkt nachzuholen.

Im Zusammenhang mit der Erarbeitung des Humanforschungsgesetzes wird diese Problematik derzeit auch in der Schweiz intensiv diskutiert. Um eine unproduktive Polarisierung der Diskussion zu vermeiden und der Komplexität der Fragestellung gerecht zu werden, lohnt es sich, konkrete Studiendesigns exemplarisch zu betrachten. In diesem Sinne werden im Folgenden ausgewählte Beispiele von Studien präsentiert und diskutiert, die verschiedene Modifikationen von einem umfassenden Informed Consent aufweisen.

\section{Was ist ein modifizierter Informed Consent?}

Kasten 1 auf der nächsten Seite zeigt die auf das Studiendesign bezogenen Details, über die die Teilnehmer üblicherweise aufgeklärt werden sollen ${ }^{a}$ [1]. Die Mitteilung von Merkmalen des Studiendesigns, wie beispielsweise die Existenz einer Vergleichsgruppe, ist in manchen Richtlinien nicht explizit gefordert, jedoch stellen diese Designmerkmale durchaus relevante Entscheidungskriterien für Patienten dar. Wird beispielsweise das Einverständnis eines Patienten zu einer Studie erfragt, bei der man die Patienten nicht verblinden kann (Raucherentwöhnungsprogramm versus kurze Raucherberatung oder keine Intervention), so können Patienten, die der Kontrollgruppe zugeteilt wurden, ihre Einwilligung zurückziehen, weil sie lieber die at-

\section{Informed Consent - Equilibre entre protection des patients et intérêts de la recherche}

L'information du patient en vue de son consentement éclairé (Informed Consent) est fermement établie comme pierre angulaire en éthique de la recherche. Ces dernières années, les débats éthiques de la recherche ont néanmoins porté en grande partie sur la question de savoir si, et dans l'affirmative, à quelles conditions il est possible d'admettre ou même de requérir des exceptions à l'exigence stricte du consentement éclairé. Alors que la recherche sur des personnes incapables de discernement a déjà fait l'objet de débats approfondis, on a jusqu'à présent beaucoup moins discuté des dérogations au consentement éclairé pour des raisons de méthode. Le présent article présente quelques cadres d'études contenant ce genre de dérogations. II entend ainsi contribuer au lancement d'une discussion éthique différenciée sur la problématique complexe d'une information lacunaire du patient pour des raisons de méthode.

traktiver erscheinende Intervention (dann eben ausserhalb der Studie) erhalten möchten.

Studien mit fehlendem oder modifiziertem Informed Consent haben in der Literatur heftige Kritik ausgelöst, insbesondere wenn ethische Prinzipien grob missachtet worden sind. Ein bekanntes, besonders eindrückliches Beispiel für Ausnahmen vom Informed Consent, die wir heute klar als missbräuchlich einordnen würden, ist die Tuskegee-Studie des Public Health Service in Alabama, in der zwischen 1932 und 1972 an fast vierhundert Schwarzen der natürliche Verlauf 
der Syphilis untersucht wurde [2]. Die Studienteilnehmer wurden weder aufgeklärt, dass sie an einer Studie teilnahmen, noch wurden sie bezüglich ihrer Erkrankung informiert. Darüber hinaus wurde ihnen bewusst kein Penicillin gegeben, das seit den 1950er Jahren erhältlich war. Solche Studien mit grober Verletzung von Persönlichkeitsrechten sollen hier nicht weiter behandelt werden. Vielmehr soll es um Studien gehen, bei

\section{Kasten 1}

Die Einholung eines detaillierten Informed Consent bedeutet, dass den Studienteilnehmern die folgenden Informationen über das Studiendesign mitgeteilt werden:

- Die Tatsache, dass es sich um eine wissenschaftliche Studie handelt

- Die Ziele der Studie

- Das Design der Studie:

- Zuteilungsmethode (Randomisierung)

- Die mit der Studie verbundenen Pflichten und Funktionen des Teilnehmers, einschliesslich Follow-up-Untersuchungen

- Umgang mit im Rahmen der Studie erhobenen Daten und Proben (Anonymisierung)

- Unterschiedliche Interventionen in den einzelnen Studienarmen

- Die Möglichkeit, eine Placebointervention oder keine Intervention zu erhalten

- Die Existenz eines anderen Studienarms, der eine attraktiver erscheinende Intervention erhält, wenn der Teilnehmer der Kontrollgruppe zugeteilt würde

- Verblindung des Teilnehmers und/oder des Arztes

- Der mögliche Nutzen und mögliche Risiken der Intervention innerhalb der Studie

- Die Tatsache, dass eine neuartige Behandlung unerwünschte Wirkungen hervorrufen kann, die nicht sicher vorhersagbar sind und die dem Teilnehmer Schaden zufügen können

- Alternative Interventionen, die ausserhalb der Studie zugänglich sind, und ihr möglicher Nutzen sowie Risiken

Neben den Einzelheiten zum Studiendesign umfasst ein detaillierter Informed Consent auch andere Aspekte wie z. B. die Freiwilligkeit der Studienteilnahme und die Möglichkeit, die Studie jederzeit ohne persönliche Nachteile abbrechen zu können oder Datenschutzmassnahmen.

\section{Kasten 2}

Kriterien von Truog et al. zum Verzicht auf einen detaillierten Informed Consent für eine randomisierte kontrollierte Studie [5]:

1. Die innerhalb der Studie angebotenen Behandlungen könnten auch ausserhalb der Studie ohne den spezifischen Informed Consent des Patienten angeboten werden.

2. Die Studienintervention sollte nicht mehr als ein minimales zusätzliches Risiko im Vergleich zu den Behandlungsalternativen beinhalten.

3. Es muss Unsicherheit über die beste Behandlung bestehen, d.h., nach dem aktuellen Wissensstand darf keine Behandlung als überlegen gelten (clinical equipoise).

4. Keine vernünftige Person sollte eine Behandlung gegenüber einer anderen bevorzugen. Das betrifft auch Unannehmlichkeiten, z.B. durch zusätzliche Arztbesuche.

5. Die Patienten sollten darüber informiert werden, dass die Institution, in der sie behandelt werden, die Punkte 1-4 berücksichtigt. denen angenommen werden kann, dass den Studienteilnehmern durch das Vorenthalten ausgewählter Informationen kein Schaden entsteht.

Im folgenden werden beispielhaft vier Studiendesigns randomisierter kontrollierter Studien beschrieben, die Ausnahmen vom detaillierten Informed Consent implizieren. Dabei ist das erste - der allgemein gehaltene Consent - bislang nur als Vorschlag in der Diskussion, die anderen Beispiele sind hingegen Teil der Forschungspraxis.

\section{Vier Beispiele für Ausnahmen vom detaillierten Informed Consent}

\section{Allgemein gehaltener Informed Consent (blanket/general consent)}

Im Rahmen einer intensiven Diskussion im British Medical Journal wurde angeregt, unter bestimmten Umständen einen allgemein gehaltenen Informed Consent zuzulassen, bei dem der Patient vor Behandlungsbeginn seine generelle Zustimmung zur Einbindung in klinische und diagnostische Studien gibt. Der betreuende Arzt müsse bestätigen, immer in guter $\mathrm{Ab}$ sicht zu handeln, und bereit sein, unkonventionelle oder neue Behandlungen zu erklären, wenn diese zu einem späteren Zeitpunkt empfohlen werden [3].

Mit diesem allgemein gehaltenen Informed Consent würden dem Patienten gewisse Informationen vorenthalten, z. B. wann genau er in eine Studie einbezogen wird und ob es eine Vergleichsgruppe gibt. Die Studiensituation und die Information für den Patienten würden der normalen Arzt-Patienten-Beziehung im Alltag sehr nahe kommen. Durch dieses Vorgehen könnten medizinische Behandlungen wissenschaftlich untersucht werden, die weithin eingesetzt werden, deren Wirksamkeit jedoch immer noch als unklar gelten muss, oder es könnte festgestellt werden, welche von verschiedenen Substanzen oder Dosierungen die überlegene ist.

Ein Beispiel aus der Praxis ist eine noch nicht vorhandene Vergleichsstudie zweier Antibiotika, die für dieselbe Indikation eingesetzt werden. Patienten, die zu Beginn ihres stationären Aufenthalts einen Generalkonsent für die Einbindung in klinische Studien gegeben hätten, würden dann ohne weitere Rückfragen randomisiert, einer der beiden Behandlungsgruppen zugeteilt und bezüglich der Intervention, die sie erhalten, aufgeklärt (z.B. zu Nebenwirkungen). Auch Brewin plädiert für eine solche allgemein gehaltene Form von Informed Consent, wobei Ethikkommissionen den Schutz der Studienteilnehmer gewährleisten sollen [4].

Truog et al. haben fünf Kriterien vorgeschlagen, nach deren Prüfung auf einen detaillierten Informed Consent des Patienten für eine randomisierte kontrollierte Studie verzichtet werden könnte (Kasten 2) [5].

Das Anliegen des Vorschlags ist nachvollziehbar. Wenn man zwei in der Praxis etablierte (zugelassene) Therapien einsetzt, genügt die Einholung des ein- 
fachen Informed Consent. Wenn man hingegen die gleichen Therapien in einer Studie vergleichen will, werden Wissenschaftler durch die gängigen Richtlinien dazu verpflichtet, über den Einschluss in eine Studie, über die randomisierte Zuteilung, über die Möglichkeit, die Einwilligung jederzeit zu widerrufen und anderes zusätzlich zu informieren. Diese ausführliche Aufklärung kann Patienten das Gefühl geben, sie würden einem Experiment ausgesetzt, das sie gefährden könnte. Tatsächlich sind die Behandlungsrisiken aber nicht höher als in der Praxis.

Es geht jedoch bei forschungsethischen Erwägungen nicht nur um das Risiko, sondern auch um den Respekt vor der Autonomie. Zwar kann es eine autonome Entscheidung darstellen, auf Informationen zu verzichten und auf das Urteil eines anderen zu vertrauen. Dies mag in der Tat unserem Vorgehen im Alltag entsprechen: «In allen Lebenslagen, in denen eine Person die Hilfe einer anderen sucht, ist das auf Vertrauen basierende Einverständnis genauso gültig wie das auf Information basierende. Es ist immer eine Mischung aus beiden, nur die Proportionen variieren.» [4] Ob das Vertrauen der Patienten jedoch so robust ist, dass sie, wenn sie die Wahl hätten, auf die Möglichkeit zu einem spezifischen Consent verzichten, ist eine offene Frage.

\section{Zelen-Designs}

Zwei andere Vorgehensweisen zur modifizierten Einholung des Informed Consent schlug der Statistiker Marvin Zelen vor. Im Jahr 1979 präsentierte er ein Studiendesign, das beim Vergleich einer experimentellen Behandlung mit einer Standardtherapie angewendet werden kann [6]. Er bezeichnete dieses Design als «single randomized consent design» oder auch als «prerandomization». Patienten, die durch die Randomisierung der experimentellen Therapie zugeteilt wurden, werden um ihr Einverständnis zu dieser Behandlung gebeten, erfahren jedoch nichts über die Tatsache der Randomisierung. Patienten, die der Standardtherapie zugeteilt wurden, erhalten bei Einverständnis die Standardtherapie, erfahren aber weder etwas über die Randomisierung noch über den Studieneinschluss.

Das «single randomized consent design» wurde nur sehr vereinzelt angewendet. Ein Grund dafür sind sicherlich ethische Bedenken wegen des Vorenthaltens von Informationen. Ein weiterer Grund ist wahrscheinlich darin zu sehen, dass dieses Studiendesign nicht mit nationalen Gesetzen vereinbar ist, die vorschreiben, dass ein Studienteilnehmer über die Tatsache informiert werden muss, dass er in einer Studie ist.

Im Jahr 1990 stellte Zelen das «double consent randomized design» vor [7]. Auch dabei erfolgt die Randomisierung vor der Einholung des Informed Consent. Einem Patienten, der der experimentellen Therapie zugeordnet wurde, schlägt man zuerst diese Therapie vor. Alle potentiellen Risiken, der Nutzen und Behandlungsalternativen werden mit ihm erör- tert. Lehnt der Patient die vorgeschlagene experimentelle Behandlung ab, wird ihm als zweites die Therapie der Kontrollgruppe oder als drittes eine weitere Alternative angeboten. Ein Patient, der der Standardtherapie zugeteilt wurde, erhält bei Einverständnis die Standardtherapie. Wenn er diese angebotene Therapie ablehnt, erhält er als zweites die experimentelle oder als drittes eine alternative Therapie vorgeschlagen. Der wesentliche Unterschied zum ersten Zelen-Design besteht darin, dass - in Übereinstimmung mit rechtlichen Vorschriften - alle Patienten gefragt werden, ob sie mit der Behandlung innerhalb einer Studie einverstanden sind. Der Prüfarzt verschweigt jedoch den Patienten die Tatsache der Randomisierung und erweckt den Eindruck, die jeweilige Behandlung sei durch professionelle Überlegungen eigens für den Patienten ausgewählt worden.

Die letztgenannten Aspekte sind in ethischer Hinsicht diskussionswürdig und sicherlich ein Grund dafür, dass das Design bisher keine weite Verbreitung und Anwendung erfahren hat. Diesen Bedenken könnte jedoch folgende Überlegung entgegengehalten werden. Wenn eine Vergleichsstudie verschiedener Therapien durchgeführt wird, besteht Ungewissheit bezüglich der Frage, welche Therapie die bessere/ beste ist. Der eine spezielle Therapie offerierende Arzt enthält dem Patienten keine als überlegen geltende Therapie vor. Die verborgene Randomisierung fügt dem Patienten keinen Schaden zu. Über die eventuell noch unbekannten Risiken der experimentellen Therapie werden die betreffenden Patienten aufgeklärt. Dennoch bleibt die Frage, ob hier dem Respekt vor der Autonomie ausreichend Genüge getan wird. Das Design käme ja insbesondere dann zum Einsatz, wenn die Rekrutierung von Patienten schwierig ist, weil ausgeprägte Präferenzen vermutet werden. Dem Patienten wird es im Falle des Zelen-Designs erschwert, seine wenngleich vor dem Massstab der evidenz-basierten Medizin nicht stichhaltigen - Präferenzen einzubringen.

\section{Einholung des Einverständnisses zum Vorenthalten von Informationen}

Ausnahmen vom detaillierten Informed Consent können auch zur Anwendung kommen, indem die Patienten zustimmen sollen, dass ihnen einige Informationen über die Behandlung im Rahmen der Studie vorenthalten werden, damit das Studienergebnis nicht verfälscht wird. Wenn Studien einen kurzfristigen Zeithorizont haben und nicht das Überleben als Zielparameter fungiert, so kann man den Patienten anbieten, die vorenthaltene Information (z. B. den Namen des Medikaments) nach Abschluss der Studie mitzuteilen. Ein Vorteil des wissentlichen Vorenthaltens von Information gegenüber dem Zelen-Design ist die Möglichkeit eines doppelblinden Vorgehens und die Möglichkeit zur Einbeziehung eines Placeboarms.

Ein Beispiel für einen «consent to postponed information» liefert eine Studie von Dennis et al. [8]. 
Die Autoren planten zu untersuchen, ob sich bei Patienten mit Schlaganfall in der Zeit nach der stationären Behandlung die Lebensqualität und die Zufriedenheit mit der Pflege unterscheidet, wenn sie eine Standardpflege oder eine weiterreichende häusliche Pflege erhalten. Die Autoren befürchteten, dass die Studienergebnisse verzerrt werden könnten, erstens weil die Patienten der Kontrollgruppe unzufrieden sein könnten, wenn sie wüssten, dass andere Patienten weiterreichende Pflege erhielten, und zweitens, weil Patienten mit weiterreichender Pflege vorteilhaftere Einschätzungen abgeben könnten, um sich gegenüber den Mitwirkenden des Projekts dankbar zu zeigen. ein Cluster, das Intervention A erhält und ein oder mehrere andere Organisationseinheiten weitere Cluster mit Vergleichsinterventionen B oder C. Die Patienten werden in der Regel nicht über die Existenz eines Vergleichsclusters aufgeklärt und haben keine individuelle Wahlmöglichkeit [10].

Ein Beispiel dafür ist die wissenschaftliche Frage, ob Patienten mit einem akuten thorakalen Syndrom (möglicher oder gesicherter Herzinfarkt) eine höhere Überlebens- und Rehabilitationsrate haben, wenn sie auf einer spezialisierten «chest unit» behandelt werden im Vergleich zur Behandlung auf einer allgemeininternistischen Station. Würde man dazu eine randomi-

\section{Es geht um eine Abwägung zwischen dem Respekt vor der Autonomie des prospektiven Studienteilnehmers und der Forschungsfreiheit bzw. dem öffentlichen Wohl}

Deshalb entschieden sich die Autoren, die Patienten nach ihrer Einwilligung zu fragen, ob sie mit vorenthaltenen Informationen einverstanden sind, die ihnen erst am Ende des Follow-up (sechs Monate nach Entlassung) mitgeteilt werden. Den Patienten wurde die Information über die Randomisierung vorenthalten, die Information über die Intervention des jeweils anderen Studienarms und die Information, dass die Studie die Effektivität des weiterreichenden Pflegeprogramms einschätzen sollte. Nach Ende der sechs Monate erhielten die Patienten einen Brief, der neben den vorenthaltenen Informationen auch die Gründe dafür enthielt.

Dieses Verfahren kann in mindestens dreierlei Hinsicht kritisiert werden. Ein erster Kritikpunkt könnte lauten, dass die Patienten nicht mit dem nötigen Respekt behandelt werden, weil sie keine informierte Entscheidung treffen können. Zweitens könnten die Patienten negative Einstellungen entwickeln gegenüber der Teilnahme an zukünftigen Forschungsprojekten. Und drittens könnte es zu einem verminderten Vertrauen in die betreuenden Ärzte kommen [9]. Als Gegenargument kann angeführt werden, dass es fragwürdig ist, teure Pflegeprogramme durchzuführen, die nicht ausreichend evaluiert wurden [9].

\section{Forschung auf dem Gebiet des öffentlichen}

\section{Gesundheitswesens - Cluster-RCTs}

Mit Hilfe von randomisierten kontrollierten Studien können nicht nur verschiedene individuelle medizinische Interventionen verglichen werden, sondern auch komplexe Interventionen, die Organisationsstrukturen im Gesundheitswesen betreffen. Das klassische Studiendesign für Forschung auf dem Gebiet des öffentlichen Gesundheitswesens ist die clusterrandomisierte kontrollierte Studie. Dabei bilden Patienten einer Organisationseinheit (z. B. Krankenhaus) sierte Studie durchführen und versuchen, den individuellen Informed Consent aller Teilnehmer einzuholen, so würden höchstwahrscheinlich kaum Patienten zustimmen, weil sie in der spezialisierten Station eine individuellere und modernere Behandlung sehen, die ihnen vorteilhafter erscheint. Die Gefahr einer «Überbehandlung» und damit eines Schadens durch invasivere Diagnose- und Behandlungsmethoden wird hierbei eher unterschätzt. Diese Studie wäre also realistisch gesehen wohl nur durchführbar, wenn man den Patienten die Tatsache der Randomisierung und einer anderen Vergleichsgruppe vorenthalten dürfte.

Cassel und Young kritisieren, dass die gegenwärtigen ethischen Richtlinien für Forschung auf dem Gebiet des öffentlichen Gesundheitswesens ungeeignet sind [11]. Diese speziellen Fragestellungen seien in den betreffenden Richtlinien nicht gesondert berücksichtigt. Ethik-Kommissionen lehnen oftmals die Genehmigung von Studien für den Vergleich unterschiedlicher Modelle für Serviceleistungen im Gesundheitswesen ab, weil die individuelle Einwilligung der Studienteilnehmer nicht vorgesehen ist [11]. Dies führt dazu, dass der potentielle Nutzen z. B. von neuen Strukturen des Gesundheitspersonals oder Screeningprogrammen nicht mit methodologisch hochwertigen Studien untersucht werden kann.

\section{Abwägung der ethischen Vertretbarkeit von Studien}

Um zu entscheiden, ob eine Studie oder ein Studiendesign mit modifiziertem Informed Consent als ethisch akzeptabel gelten kann oder nicht, ist es wichtig, den gesamten Kontext zu betrachten. Auf der einen Seite sind der Grad der Beeinträchtigung des Selbstbestimmungsrechts der Patienten und das Ausmass seiner Belastung durch die Studie auszumachen. Auf der anderen Seite ist die Bedeutsamkeit der betref- 
fenden Forschungsfrage vor dem Hintergrund möglicher alternativer Studiendesigns inklusive ihrer Vorund Nachteile zu diskutieren. Es geht also um eine Abwägung zwischen dem Respekt vor der Autonomie des prospektiven Studienteilnehmers und der Forschungsfreiheit bzw. dem öffentlichen Wohl.

Dabei ist auf die Vereinbarkeit mit der nationalen Gesetzgebung zu achten. In vielen Ländern ist die informierte Einwilligung zwar Teil der Gesetzgebung, die detaillierte Ausgestaltung jedoch eine Auslegungssache. Zum Beispiel ist in Deutschland die Aufklärung eines Studienpatienten über die Tatsache der randomisierten Zuteilung der Patienten zu den Behandlungen und über die Existenz anderer Vergleichsgruppen nicht explizit in den Gesetzestexten abgebildet [12].

In der Schweiz wird derzeit darüber debattiert, inwieweit das künftige Humanforschungsgesetz Ausnahmen vom detaillierten Informed Consent gestatten soll [13]. In Art. 18 des Entwurfs eines Bundesgesetzes über die Forschung am Menschen vom 21. Oktober 2009, der nunmehr zur Beratung ans Parlament überwiesen wurde, finden sich Bestimmungen zur unvollständigen Aufklärung, die auf eine Balance zwischen dem Schutz der Forschungsteilnehmer und den Interessen der Forschung zielen. Der Artikel sieht vor, dass Personen ausnahmsweise über einzelne Punkte eines Forschungsprojekts vor dessen Beginn unvollständig aufgeklärt werden dürfen, wenn dies aus methodischen Gründen zwingend ist und wenn mit dem Forschungsprojekt nur minimale Risiken und Belastungen verbunden sind. Die teilnehmende Person muss nachträglich so bald als möglich hinreichend aufgeklärt werden und kann sodann entscheiden, ob sie in die Verwendung ihrer Daten oder ihres biologischen Materials zu den beschriebenen Zwecken einwilligt ${ }^{\mathrm{b}}$. Risiko und Belastung für die Probanden sind also explizit eingeschränkt. Der Respekt vor der Autonomie könnte noch durch das folgende, im Artikel nicht explizite Erfordernis gestärkt werden: Die Probanden sollten vor Beginn der Studie, wenn sie um ihre Einwilligung gebeten werden, über die Tatsache informiert werden, dass ihnen bestimmte Aspekte der Studie aus methodischen Gründen vorenthalten werden. Sie sollten auch wissen, dass sie im Nachhinein vollständig aufgeklärt werden und das Recht haben werden, über die Verwendung ihrer Daten bzw. ihres biologischen Materials zu entscheiden. Unter diesen Voraussetzungen können die prospektiven Studienteilnehmer selbst entscheiden, ob sie angesichts der minimalen Belastung bzw. des minimalen Risikos diese Ungewissheit eingehen möchten.

Mit dem Gesetzesentwurf ist ein vielversprechender Anfang für die notwendige differenzierte Debatte gemacht. Dabei geht es darum, konsistente und argu- mentativ plausibel begründete Positionen zu entwickeln, was die Kenntnis wichtiger methodologischer Zusammenhänge und Studiendesigns voraussetzt. Hierfür wäre eine vergleichende Zusammenschau und Beurteilung relevanter Studiendesigns ein notwendiger nächster Schritt. Wenn es um die diffizile Balance zwischen Patientenschutz und Forschungsinteressen geht, ist es wichtig, Klarheit und Sicherheit zu schaffen, für Studienteilnehmer wie für Forschende.

\section{Literatur}

1 Fässler M, Biller-Andorno N. Die Einholung des Informed Consent - ein bisher nicht ausreichend berücksichtigtes Validitätskriterium von randomisierten Therapiestudien. Poster bei der 6. Jahrestagung des DNEbM. EbM. 2005. Berlin; 2005.

2 Jones JH. Bad Blood: The Tuskegee Syphilis Experiment. New York: Free Press; 1993.

3 Tobias JS. BMJ's present policy (sometimes approving research in which patients have not given fully informed consent) is wholly correct. BMJ. 1997;314(7087):1111-4.

4 Brewin T. Informed consent. «Blanket» consent to trials would be a good idea. Letter. BMJ. 1997;315(7102):253.

5 Truog RD, Robinson W, Randolph A, Morris A. Is informed consent always necessary for randomized, controlled trials? N Engl J Med. 1999;340:804-7.

6 Zelen M. A New Design for Randomized Clinical Trials. N Engl J Med. 1979;300(22):1242-5.

7 Zelen M. Randomized consent designs for clinical trials: an update. Stat in Med. 1990;9:645-56.

8 Dennis M, O'Rourke S, Slattery J, Staniforth T, Warlow C. Evaluation of a stroke family care worker: results of a randomised controlled trial. BMJ. 1997;314:1071-7.

9 Boter H, van Delden JJ, de Haan RJ, Rinkel GJ. Modified informed consent procedure: consent to postponed information. BMJ. 2003;327(7409):284-5.

10 Chenot JF. Cluster-randomisierte Studien: eine wichtige Methode in der allgemeinmedizinischen Forschung. Z Evid Fortbild Qual Gesundhwes. 2009;103(7):475-80.

11 Cassell J, Young A. Why we should not seek individual informed consent for participation in health services research. J Med Ethics. 2002;28(5):313-7.

12 Andorno R, Fässler M, Biller-Andorno N. Can there be exceptions to fully informed consent in randomized clinical trials? The case of the randomized consent (Zelen) design. Annual Review of Law and Ethics. 2007;15:339-56.

13 Biller-Andorno N, Fässler M. Auch eine irreführende Aufklärung kann akzeptabel sein. Neue Zürcher Zeitung 11.9.2008, S. 18. am 4. November 2009. 00702/07558/index.
html?lang=de, letzter Zugriff

b Vgl. www.bag.admin.ch/ themen/medizin/00701/ 